\title{
Inhibition of GSK3 and MEK induced cancer stem cell generation via the Wnt and MEK signaling pathways
}

\author{
SHENGTAO LIAO $^{1}$, LI GAN $^{2}$, WANXIANG QIN ${ }^{3}$, CHANG LIU $^{4}$ and ZHECHUAN MEI ${ }^{1}$ \\ ${ }^{1}$ Department of Gastroenterology, The Second Affiliated Hospital of Chongqing Medical University, \\ Chongqing 400010; ${ }^{2}$ Teaching and Research Section of Forensic Medicine, College of Basic Medicine, \\ Chongqing Medical University, Chongqing 400016; ${ }^{3}$ Department of Pain Management, Southwest Hospital, \\ The First Affiliated Hospital of The Third Military Medical University; ${ }^{4}$ Institute of Medicine \\ and Hygienic Equipment for High Altitude Region, College of High Altitude Military Medicine, \\ Third Military Medical University, Chongqing 400038, P.R. China
}

Received November 30, 2017; Accepted July 17, 2018

DOI: 10.3892/or.2018.6600

\begin{abstract}
Cancer stem cells (CSCs) are considered to be tumor-initiating cells, responsible for tumor invasive growth and dissemination to distant organ sites. Typically, radiation treatment and chemotherapy should target CSCs. However, current research investigating CSCs is impeded by the difficulty of isolating pure CSCs and maintaining them in vitro. In the present study, the synergistic inhibition of glycogen synthase kinase 3 and mitogen-activated protein kinase kinase using small molecules, CHIR99021 and PD184352, efficiently generated CSCs from immortalized human mammary epithelial cells (HMLEs) and resulted in the acquisition of mesenchymal traits and the expression of epithelial-mesenchymal transition markers. The cell proliferation, invasion and migration of HMLE cells were significantly promoted by CHIR99021 and PD184352 $(\mathrm{P}<0.05)$. Furthermore, the cell cycle was shifted from the G0/G1 phase to the G2/M phase, and the apoptotic rate was suppressed in HMLE cells following treatment with CHIR99021 and PD184352. Compared with control group, the stimulated cells exhibited an increased ability to form mammospheres and regenerate a tumor. In addition to these properties, the induced cells also exhibited notable chemotherapy resistance. In vivo, the treatment of cells with CHIR99021 and PD184352 promoted the growth of HMLE-engrafted tumor types. These results provide a practical strategy for the generation of CSCs using small molecules in vitro, which provides a cell resource that may be used for drug screening. Additionally, the present results additionally highlighted the synergistic functions of
\end{abstract}

Correspondence to: Dr Zhechuan Mei, Department of Gastroenterology, The Second Affiliated Hospital of Chongqing Medical University, 76 Linjiang Road, Chongqing 400010, P.R. China E-mail: zhechuanmei@126.com

Key words: cancer stem cell, epithelial-mesenchymal transition, mitogen-activated protein kinase kinase, glycogen synthase kinase 3
Wnt and mitogen-activated protein kinase kinase signaling pathways in tumorigenesis.

\section{Introduction}

Accumulating evidence suggests that different cancer types are hierarchically organized and only a small fraction of all tumor cells are essential for tumor initiation, invasive growth and potential dissemination to distant organ sites, through blood or lymphatic vessels (1). These few cells, termed 'cancer stem cells' (CSCs), possess an enhanced self-renewal capacity and the ability to differentiate into multiple lineages $(2,3)$. Although certain properties and functions of CSCs have been explored, further in-depth research is impeded by the difficulty of isolating pure CSCs and maintaining them in vitro (4).

Despite numerous attempts, there remains a lack of robust methods able to isolate CSCs. Previously, certain studies have experienced difficulty using markers that originally appeared to accurately distinguish tumorigenic from non-tumorigenic cells (5-7). Therefore, the surface markers that have been used to isolate CSCs may be unreliable.

In an attempt to generate CSCs from normal cancer cells using small molecules, a homogenously converted cell population was generated by combined treatment with two small molecules, CHIR99021 and PD184352 (5). The cells exhibited properties similar to CSCs, including a self-renewal capacity and stability in maintaining CSC characteristics. As an inhibitor of glycogen synthase kinase 3 (GSK3), CHIR99021 (referred to as CHIR) is implicated in the self-renewal of embryonic stem cells, activating canonical Wnt signaling (8,9). PD184352 (referred to as PD) is a small inhibitor of mitogen-activated protein kinase kinase (MEK) that has been demonstrated to suppress cell proliferation (10). Subsequent studies reported that the expression levels of certain proteins that notably mediate migration and invasion change during the process of epithelial-mesenchymal transition (EMT) $(11,12)$.

This novel method provides a practical strategy to generate CSCs, which may be subsequently used for small molecule drug screening in vitro. The mechanisms that mediated the induction of CSCs remain largely unknown and require further 
investigation. The aim of the present study was to investigate the effect of the inhibition of GSK3 and MEK in CSCs and the underlying molecular mechanisms.

\section{Materials and methods}

Cell culture. Immortalized human mammary epithelial cells (HMLE; Type Culture Collection of the Chinese Academy of Sciences, Shanghai, China) were maintained in Dulbecco's modified Eagle's medium (DMEM; Thermo Fisher Scientific, Inc., Waltham, MA, USA): F12 media (Thermo Fisher Scientific,Inc.) (1:1) supplemented with insulin (Sigma-Aldrich; Merck KGaA, Darmstadt, Germany), epidermal growth factor, hydrocortisone (Sigma-Aldrich; Merck KGaA) and 5\% fetal bovine serum (FBS; GE Healthcare Life Sciences, Logan, UT, USA) at $37^{\circ} \mathrm{C}$ in a humidified atmosphere containing $5 \% \mathrm{CO}_{2}$. The cells were passaged every 3-5 days. The HMLE cells were treated with $3 \mu \mathrm{M}$ PD (Meiyan Biological Technology Co., Ltd, Shanghai, China) and $2 \mu \mathrm{M}$ CHIR (Wako Pure Chemicals Industries, Ltd., Osaka, Japan). Cultured cells were imaged using an inverted confocal laser scanning microscope (TCS SP5; Leica Microsystems GmbH, Wetzlar, Germany) on day 3 at a magnification of $x 40-200$. In brief, single cells were transferred to $50 \%$ Matrigel and cultured in mammary epithelial cell growth medium (Clonetics; Lonza Group, Ltd., Basel, Switzerland) supplemented with $10 \mathrm{ng} / \mathrm{ml}$ basic fibroblast growth factor for an additional 7 days at $37^{\circ} \mathrm{C}$. The structures were then imaged using an inverted confocal laser scanning microscope (TCS SP5; Leica Microsystems GmbH) at a magnification of $\times 40-200$.

Immunocytochemical analysis. HMLE cells were washed once with PBS (Invitrogen; Thermo Fisher Scientific, Inc.; without $\mathrm{Ca}^{2+}$ and $\mathrm{Mg}^{2+}$ ) and were fixed with a $4 \%$ formaldehyde solution containing $0.15 \%$ picric acid (Sigma-Aldrich; Merck KGaA) in PBS for $20 \mathrm{~min}$ at $37^{\circ} \mathrm{C}$, followed by three washes with PBS. Blocking and permeabilization were performed using 10\% donkey serum (Jackson ImmunoResearch Laboratories, Inc., West Grove, PA, USA) and 0.3\% Triton X-100 (Sigma-Aldrich; Merck KGaA) solution in PBS for $1 \mathrm{~h}$ at room temperature. All primary antibodies (mouse polyclonal anti-E-cadherin and anti-vimentin; dilution, 1:1,000; catalog nos. 3195 and 3932, respectively; Cell Signaling Technology, Inc., Danvers, MA, USA) were diluted in $1 \%$ bovine serum albumin (BSA) and incubated overnight at $4^{\circ} \mathrm{C}$. After $1 \mathrm{~h}$ of washing with $0.1 \%$ BSA in PBS, samples were incubated with Alexa 555- or Alexa 488-conjugated anti-mouse secondary antibodies (dilution, 1:500; cat nos. P0190 and P0188, respectively; Beyotime Institute of Biotechnology, Haimen, China) for $1 \mathrm{~h}$ at room temperature and nuclei were stained with DAPI (100 $\mu$ l; dilution, 1:1,000; Sigma-Aldrich; Merck KGaA) at room temperature for 2-3 min. All images were captured using a Nikon Eclipse 80i fluorescence microscope equipped with a PhotometricsCoolSnap HQ2 camera at a magnification of $x 40$ and processed with NIS Elements Basic Research Software 6.0 (Nikon Corporation, Tokyo, Japan).

Cell proliferation assay. The proliferative activity of HMLE cells was measured using a trypan blue dye exclusion assay. Cells were seeded in a 96-well plate at a density of 5,000 cells/well.
After $12 \mathrm{~h}, 3 \mu \mathrm{M}$ PD and $2 \mu \mathrm{M}$ CHIR were applied to the cells for 24, 48, 72 and $96 \mathrm{~h}$ and control cells were treated with the same volume of $\mathrm{PBS}$ at $37^{\circ} \mathrm{C}$. In order to investigate whether cells treated with CHIR and PD exhibited CSC properties, cells were treated with serial dilutions of doxorubicin $(100,200$ and 400 ng/ml; Lingnan Pharmaceutical, Ltd., Guangzhou, China) or paclitaxel (10, 20 and $40 \mathrm{ng} / \mathrm{ml}$; Lingnan Pharmaceutical, Ltd., Guangzhou, China) for $24 \mathrm{~h}$ at $37^{\circ} \mathrm{C}$ and control cells were treated with dimethyl sulfoxide $(0.2 \%$; Sigma-Aldrich; Merck $\mathrm{KGaA}$ ). The cells were then trypsinized and re-suspended in PBS. Trypan blue dye solution (0.4\%) was added to the cell suspension for $2 \mathrm{~min}$ at $37^{\circ} \mathrm{C}$. After $2 \mathrm{~min}$, the number of stained (dead) cells and unstained (viable) cells per $\mathrm{mm}^{2}$ was counted under a phase contrast microscope at a magnification of $\mathrm{x} 100$.

Cell invasion and migration assays. For the invasion and migration assays, $1 \times 10^{5}$ cells/ml HMLE cells were used following treatment with CHIR and PD. The cell migration and invasion capacity were determined using a Transwell assay (Corning Incorporated, Corning, NY, USA). Cells were resuspended in serum-free DMEM medium (Thermo Fisher Scientific, Inc.). Subsequently, $200 \mu 1$ cell suspensions were seeded into the upper chamber with a porous membrane coated with Matrigel (BD Biosciences, San Jose, CA, USA; for the Transwell invasion assay) or without coating (for the migration assay). Complete DMEM medium (Thermo Fisher Scientific, Inc.) was added to the lower wells of the chambers. Once cells were allowed to migrate for $24 \mathrm{~h}$ or invade for $48 \mathrm{~h}$, cells on the upper membrane were removed using a cotton swab, and those that had migrated or invaded to the bottom of the inserts were stained with crystal violet $(1 \mathrm{mg} / \mathrm{ml}$; Sigma-Aldrich; Merck $\mathrm{KGaA}$ ) for $15 \mathrm{~min}$ at room temperature, and then the number of migratory and invasive cells were counted in five randomly selected high-power fields under a light microscope (magnification, x40; TS100; Nikon Corporation). The presented data represent three individual wells.

Reverse transcription-quantitative polymerase chain reaction (RT-qPCR) analysis. Total RNA was extracted from HMLE cells following treatment with CHIR and PD using the RNeasy Plus mini kit with QiaShredder columns (Qiagen $\mathrm{GmbH}$, Hilden, Germany) according to the manufacturer's protocol. Total RNA $(1 \mu \mathrm{g})$ per sample was reverse transcribed using the iScriptcDNA synthesis kit (Bio-Rad Laboratories, Inc., Hercules, CA, USA) according to the manufacturer's protocol and the cDNA was diluted with $100 \mu 1$ water. A total of $1 / 50$ of the diluted cDNA was used for qPCR with iQ SYBR Green Supermix on the CFX96 system (Bio-Rad Laboratories, Inc.). The PCR conditions were as follows: Initial reaction at $42^{\circ} \mathrm{C}$ for $1 \mathrm{~h}$ was used for cDNA synthesis, followed by denaturation at $94^{\circ} \mathrm{C}$ for $5 \mathrm{~min}$ and 22 cycles of the following reactions: $94^{\circ} \mathrm{C}$ for $30 \mathrm{sec}, 55^{\circ} \mathrm{C}$ for $30 \mathrm{sec}$ and $72^{\circ} \mathrm{C}$ for $30 \mathrm{sec}$. Following the last cycle, the reaction was amplified at $72^{\circ} \mathrm{C}$ for $10 \mathrm{~min}$. All qPCR reactions were performed in triplicate, and expression levels were analyzed using CFX manager software (version 3.1; Bio-Rad Laboratories, Inc.), with levels normalized to GAPDH. The expression levels of the genes were determined using the $\Delta \Delta \mathrm{Cq}$ method (13). The following primer pairs were used: E-cadherin forward, 5'-CAATGGTGTCCATGT GAACA-3' and reverse, 5'-CCTCCTACCCTCCTGTTCG-3'; 
Vimentin forward, 5'-CGCTTCGCCAACTACAT-3' and reverse, 5'-AGGGCATCCACTTCACAG-3'; Twist forward, 5'-AGCAACAGCGAGGAAGAGCC-3' and reverse, 5'-CAC AGCCCGCAGACTTCTTG-3'; $\alpha$-SMA forward, 5'-TCCCTT GAGAAGAGTTACGAGTTG-3' and reverse, 5'-ATGATG CTGTTGTAGGTGGTTTC-3' and GAPDH forward, 5'-AAT GGGCAGCCGTTAGGAAA-3' and reverse, 5'-GCCCAATAC GACCAAATCAGAG-3'. Each set of reactions was repeated using cDNA from at least three independent experiments.

Cell cycle analysis and apoptosis analysis. HMLE cells were seeded into 6-well plates at a density of $3 \times 10^{5}$ cells/well and treated with $3 \mu \mathrm{M}$ PD and $2 \mu \mathrm{M}$ CHIR for $48 \mathrm{~h}$ at $37^{\circ} \mathrm{C}$. Subsequently, cells were collected with low-speed centrifugation $\left(300 \mathrm{x} \mathrm{g}, 5 \mathrm{~min}, 4^{\circ} \mathrm{C}\right)$ and cell pellets were re-suspended in $1 \mathrm{ml}$ PBS solution. Prior to flow cytometry analysis, cells were washed, centrifuged $\left(300 \mathrm{x} \mathrm{g}, 5 \mathrm{~min}, 4^{\circ} \mathrm{C}\right)$ and re-suspended in propidium iodide (PI; Sigma-Aldrich; Merck KGaA) staining buffer containing $50 \mu \mathrm{l} / \mathrm{ml} \mathrm{PI}$ and $250 \mu \mathrm{l} / \mathrm{ml}$ RNase A at $4^{\circ} \mathrm{C}$ for $30 \mathrm{~min}$. Finally, the cell mixture was incubated at $4^{\circ} \mathrm{C}$ for $30 \mathrm{~min}$ in the dark environment to detect cell cycle and stained with $5 \mu \mathrm{l}$ Annexin V-fluorescein isothiocyanate at room temperature for $15 \mathrm{~min}$ to detect apoptosis by fluorescence activated cell sorting (FACS) analysis (Beckman Coulter, Inc., Brea, CA, USA). Apoptotic cells were analyzed using a flow cytometer and FlowJo software (version 10; FlowJo LLC, Ashland, OR, USA).

FACS analysis. Cells were washed with PBS and dissociated with Accutase (Innovative Cell Technologies, Inc., San Diego, CA, USA). Following harvesting, the cells were washed twice with ice-cold FACS buffer (Hank's balanced salt solution supplemented with $10 \mathrm{mM}$ HEPES, $2 \%$ FBS and $0.1 \%$ sodium azide; Sigma-Aldrich; Merck KGaA). Non-dissociated cells were removed by passing the cell suspension through a cell strainer (BD Biosciences) twice. Cells were incubated with phycoerythrin-conjugated anti-human CD24 antibody and allophycocyanin-conjugated anti-human CD44 antibody (dilution, 1:50; cat nos. MA5-11833 and MA5-13890, respectively; Thermo Fisher Scientific, Inc.) for $30 \mathrm{~min}$ at $4^{\circ} \mathrm{C}$. Following incubation, the cells were washed twice with 2 ml FACS buffer, fixed for $15 \mathrm{~min}$ at room temperature and suspended in 4\% paraformaldehyde solution (Electron Microscopy Sciences, Hatfield, PA, USA) in PBS. Finally, $>20,000$ cells were analyzed using FACS Calibur and CellQuest software (version 5.1; BD Biosciences). Further analysis was performed using FlowJo software (version 10; FlowJo LLC). Expression of CD24 and CD44 was quantified in divided cells and separated into 'high' and 'low' expression groups as previous described (14).

Animal studies. A total of 132 female SCID mice (weight, $16.83 \pm 0.65 \mathrm{~g}$; age, 4 weeks old; obtained from the Experimental Animal Centre of the Third Military Medical University, Chongqing, China) were maintained under specific pathogen-free conditions with 12-h light/12-h dark cycles at $26-28^{\circ} \mathrm{C}$ and $50-65 \%$ humidity, with ad libitum access to food and water. HMLE cells $\left(1 \times 10^{3}, 1 \times 10^{4}, 1 \times 10^{5}\right.$ and $1 \times 10^{6}$ cells in each group) were subcutaneously injected into the left flank of SCID mice ( $\mathrm{n}=12$ animals/group). Every 7 days post-inocula- tion, the length and width of the individual orthotopic tumors were measured with calipers, and the volume $\left(\mathrm{mm}^{3}\right)$ was calculated according to the following formula: $1 / 2 x$ length $x$ width $^{2}$. The mice were sacrificed at 42 days post-inoculation. Subcutaneous tumor growth was measured for 42 days following inoculation. Mouse subcutaneous tumors were harvested and weighed. All animal experiments were ethically approved by the Research Ethics Committee of Third Military Medical University. Mice were anaesthetized using 2\% pentobarbital sodium (0.1 ml/100 g; Sigma-Aldrich; Merck KGaA) and cervical vertebrae were dislocated.

Western blot assay. Cells were lysed in a lysis buffer containing aprotinin, leupeptin and phenylmethanesulfonyl fluoride (Sigma-Aldrich; Merck KGaA) and phosphatase inhibitor cocktails II and III (Sigma-Aldrich; Merck KGaA) at $4^{\circ} \mathrm{C}$ for $30 \mathrm{~min}$. Protein concentration was quantified using the Bradford method (15). Subsequently, $50 \mathrm{mg}$ total protein extracts were separated by $10 \%$ SDS-PAGE and transferred to polyvinylidene difluoride membranes (GE Healthcare, Chicago, IL, USA), followed by blocking for $1 \mathrm{~h}$ at room temperature in blocking buffer (cat no. P0023B; Beyotime Institute of Biotechnology). The membrane was incubated with the following primary antibodies overnight at $4^{\circ} \mathrm{C}$ : Rabbit anti-phosphorylated (p)-MEK1/2, rabbit anti-p-ERK1/2, rabbit anti- $\beta$-catenin, rabbit anti-p-GSK3 and rabbit anti-anti- $\beta$-actin antibody (dilution, 1:1,000; cat nos. 8727, 4376, 8480, 9323 and 4970, respectively; Cell Signaling Technology, Inc.). Membranes were then washed twice with PBS with Tween-20 (0.1\%). Subsequently they were incubated with horseradish peroxidase-conjugated anti-rabbit secondary antibody (dilution, 1:10,000; cat no. 7074; Cell Signaling Technology, Inc.) for $1 \mathrm{~h}$ at room temperature. Binding of the primary antibody was detected using an enhanced chemiluminescence kit (GE Healthcare). ImageJ software version 1.47 (National Institutes of Health, Bethesda, MD, USA) was used to analyze relative protein band density. Each sample was analyzed in triplicate.

Statistical analysis. All data are presented as mean \pm standard deviation except where stated otherwise. All statistical analyses were performed using SPSS 18.0 version (SPSS, Inc. Chicago, IL, USA). An unpaired Student's t-test was used to compare between the two groups. $\mathrm{P}<0.05$ was considered to indicate a statistically significant difference.

\section{Results}

$P D$ and CHIR induces mesenchymal morphological transformation and proliferation of HMLE cells. To determine whether PD and CHIR are able to induce CSCs in vitro, the morphology of HMLE cells was observed following treatment with PD and CHIR. The untreated cells exhibited oval or cobblestone shape with tight intercellular junctions (Fig. 1A; left panel). Following PD and CHIR treatment for 3 days, the cell contact between cells was lost and an elongated fibroblastic morphology was observed (Fig. 1A; right panel). There was no significant difference in proliferation between the two groups at $48 \mathrm{~h}$. Subsequently, the treated cells proliferated significantly faster compared with the control cells at 72 and $96 \mathrm{~h}(\mathrm{P}<0.05$; Fig. 1B), which was consistent with the results 


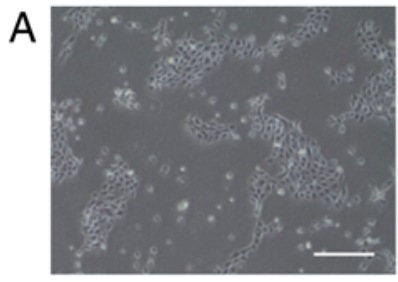

Control

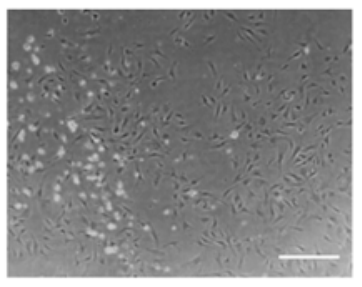

$\mathrm{PD}+\mathrm{CHIR}$

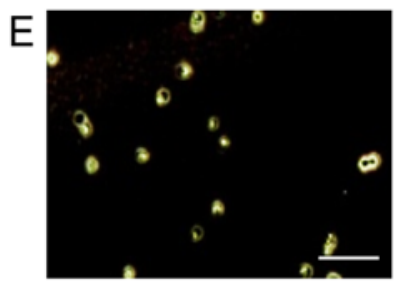

Control

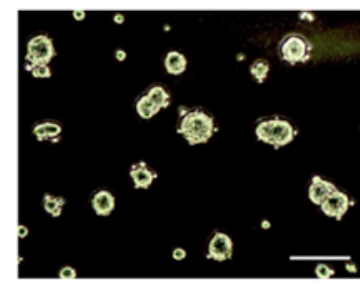

$\mathrm{PD}+\mathrm{CHIR}$
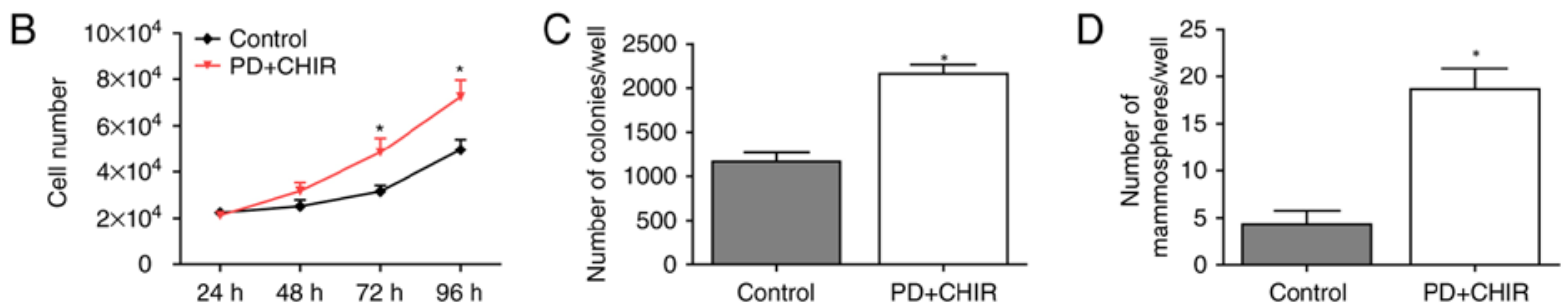

Figure 1. Morphology and proliferation of cancer cells following treatment with compounds. (A) Left panel: Representative micrographs presented an oval or cobblestone-like appearance of normal human mammary epithelial cells. Right panel: A slight increase in cell size compared with the control cells, accompanied with cell flattening, a partial loss of cell-cell adhesion and the formation of the spindle-like cells, following stimulation with compounds for 3 days. Scale bar, $100 \mu \mathrm{m}$. (B) Viable cells were counted using trypan blue dye exclusion at 24, 48, 72 and $96 \mathrm{~h}$. (C) Colony numbers were counted in each well. (D) Quantification of the mammospheres formed by cells from the control and treated cell populations. (E) Phase-contrast images of mammospheres of control and the treated cells. All assays were performed in duplicates and data are presented as the mean \pm standard deviation. ${ }^{*} \mathrm{P}<0.05$ vs. control group. PD, PD184352; CHIR, CHIR99021.
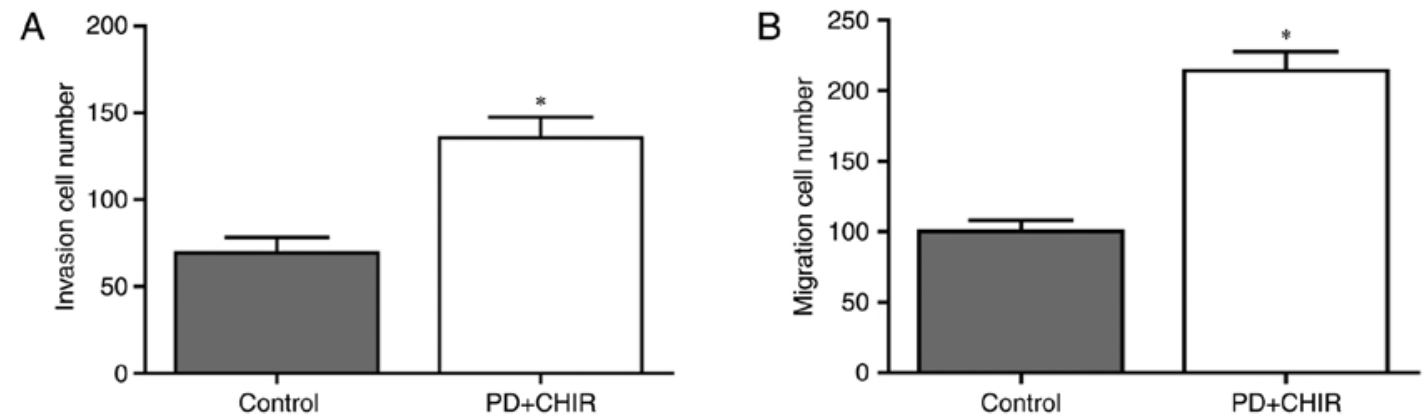

Figure 2. Cell invasion and migration of HMLE cells are promoted following treatment with CHIR and PD. (A) An invasion assay was used to determine the invasion activity of HMLE cells following treatment with CHIR and PD. (B) A migration assay was used to determine the migration activity of HMLE cells following treatment with CHIR and PD. All assays were performed in duplicate and data are presented as the mean \pm standard deviation. ${ }^{*} \mathrm{P}<0.05$ vs. control group. HMLE, human mammary epithelial cells; PD, PD184352; CHIR, CHIR99021.

of a previous study that identified that E-cadherin knockdown generates CSCs (16). Additionally, there was a significantly increased number of colonies in the treated cells compared with the control cells $(\mathrm{P}<0.05$; Fig. 1C). Furthermore, HMLE cells were seeded at a very low density in plates containing serum-free medium with or without the compounds, and the number of newly formed mammospheres was determined. Compared with the control cells, the treated cells generated a significantly greater number of mammospheres, and they were larger in size $(\mathrm{P}<0.05$; Fig. 1D). As presented in Fig. 1E, the treated cells were able to form 20 spheres per 1,000 cells while the control cells formed only 2 spheres per 1,000 cells.

Cell invasion and migration in HMLE are promoted following treatment with CHIR and PD. To determine whether treatment with CHIR and PD promoted migration and invasion potential, HMLE cells were treated with CHIR and PD, and the invasion and migration of cells were analyzed. CHIR and PD significantly promoted invasion $(\mathrm{P}<0.05$; Fig. $2 \mathrm{~A})$ and migration $(\mathrm{P}<0.05$; Fig. $2 \mathrm{~B})$ in HMLE cells compared with the control group. These data suggested that HMLE cells treated with CHIR and PD exhibited increased motility.

Cell cycle is accelerated and the apoptosis rate is suppressed in HMLE cells treated with CHIR and PD. The cell cycle distribution and apoptosis rate in HMLE cells following treatment with CHIR and PD were determined using flow cytometry analysis. These data suggested that the cell cycle prominently shifted from G0/G1 phase to $\mathrm{G} 2 / \mathrm{M}$ phase; the percentage of cells in G0/G1 phase significantly decreased and the percentage in $\mathrm{S}$ phase significantly increased compared with the control cells $(\mathrm{P}<0.05)$. Furthermore, cell apoptosis was significantly suppressed by treatment with CHIR and PD $(\mathrm{P}<0.05$; Fig. 3A). Additionally, the present study observed that the treatment of HMLE cells with the aforementioned compounds induced a shift in CD44 and CD24 expression, from $\mathrm{CD} 44^{+} / \mathrm{CD} 24^{+}$to $\mathrm{CD} 44^{\text {high }} \mathrm{CD} 24^{\text {low }}$ (Fig. $3 \mathrm{~B}$ ).

Cells treated with CHIR and PD express EMT markers. To determine whether the morphological transformation was 

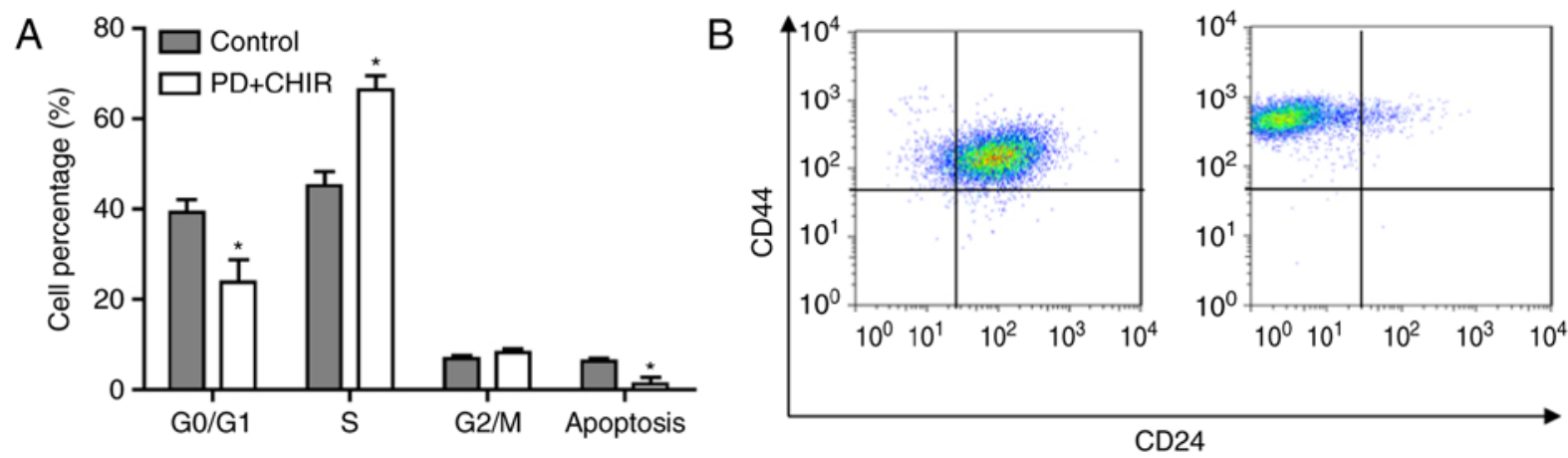

Figure 3. Cell cycle is accelerated and the apoptosis rate is suppressed by CHIR and PD in HMLE cells. (A) Cell cycle distribution and apoptosis rate in HMLE cells following treatment with CHIR and PD were determined using flow cytometry analysis. (B) Flow cytometry analysis of CD44 and CD24 for control cells, and cells treated with CHIR and PD. All assays were performed in duplicates and data are presented as the mean \pm standard deviation. ${ }^{*} \mathrm{P}<0.05$ vs. control group. HMLE, human mammary epithelial cells; PD, PD184352; CHIR, CHIR99021.

A

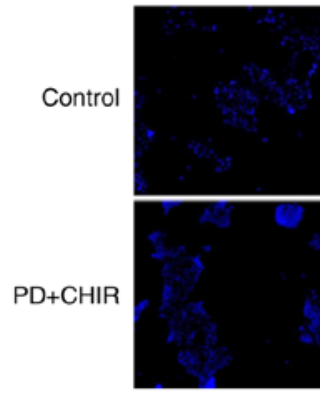

DAPI

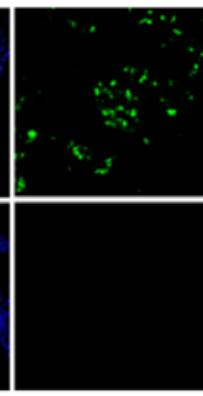

E-cadherin

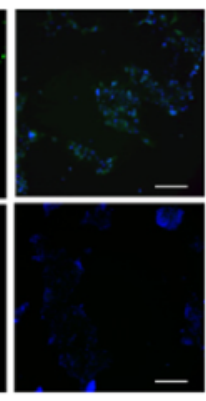

Merged

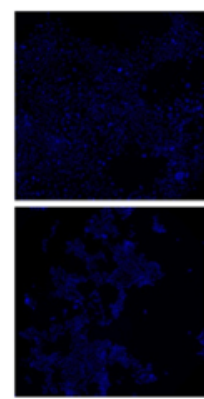

DAPI

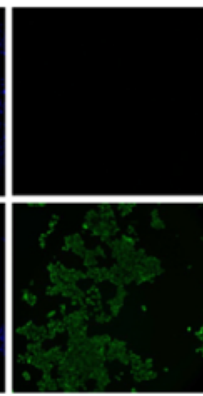

Vimentin

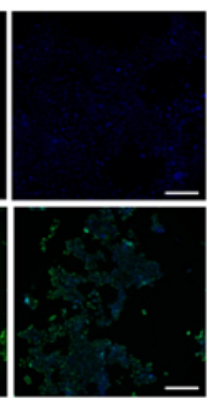

Merged
B

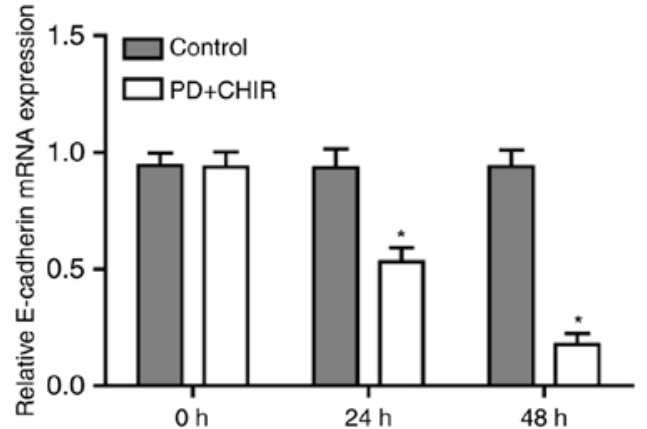

D

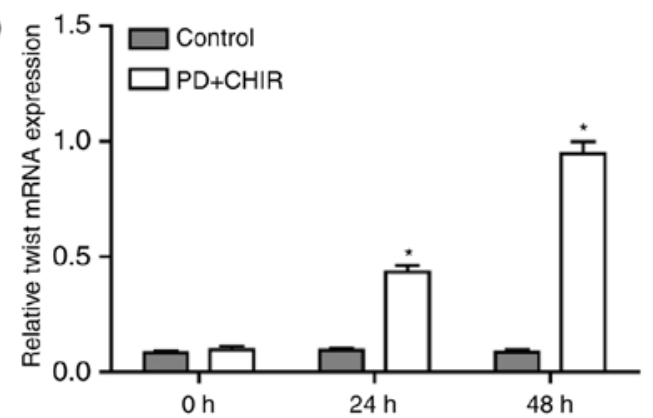

C

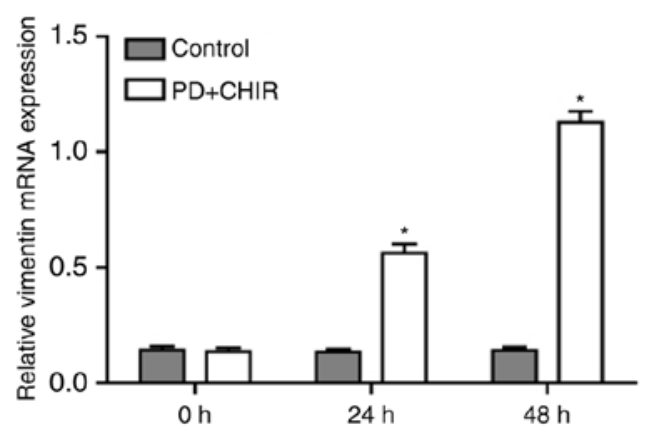

E

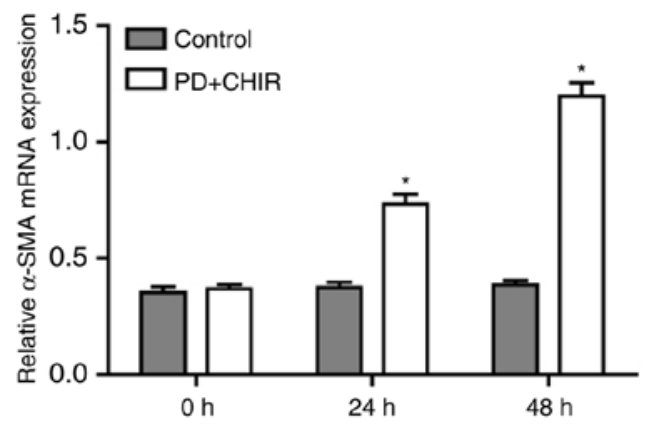

Figure 4. Epithelial-mesenchymal transition markers are increased in cells treated with CHIR and PD. (A) Immunofluorescence images of cells stained using antibodies against E-cadherin and vimentin, and nuclei were stained using DAPI. mRNA expression levels of (B) E-cadherin, (C) vimentin, (D) Twist and (E) $\alpha$-SMA measured by reverse transcription-quantitative polymerase chain reaction analysis at 0,24 and $48 \mathrm{~h}$ following treatment. All assays were performed in duplicates and data are presented as the mean \pm standard deviation. ${ }^{*} \mathrm{P}<0.05$ vs. control group. PD, PD184352; CHIR, CHIR99021; $\alpha$-SMA, $\alpha$-smooth muscle actin.

associated with the phenotypic switch, the expression of specific epithelial marker E-cadherin and the stem cell specific marker Vimentin were determined following treatment of HMLE cells with PD and CHIR. The mRNA expression levels of E-cadherin mRNA were significantly decreased following the CHIR and PD treatments at 24 and $48 \mathrm{~h}$ compared with the control cells, and the expression levels almost undetectable after $48 \mathrm{~h}$ of treatment ( $\mathrm{P}<0.05$; Fig. $4 \mathrm{~A}$ and B; left panel). By 

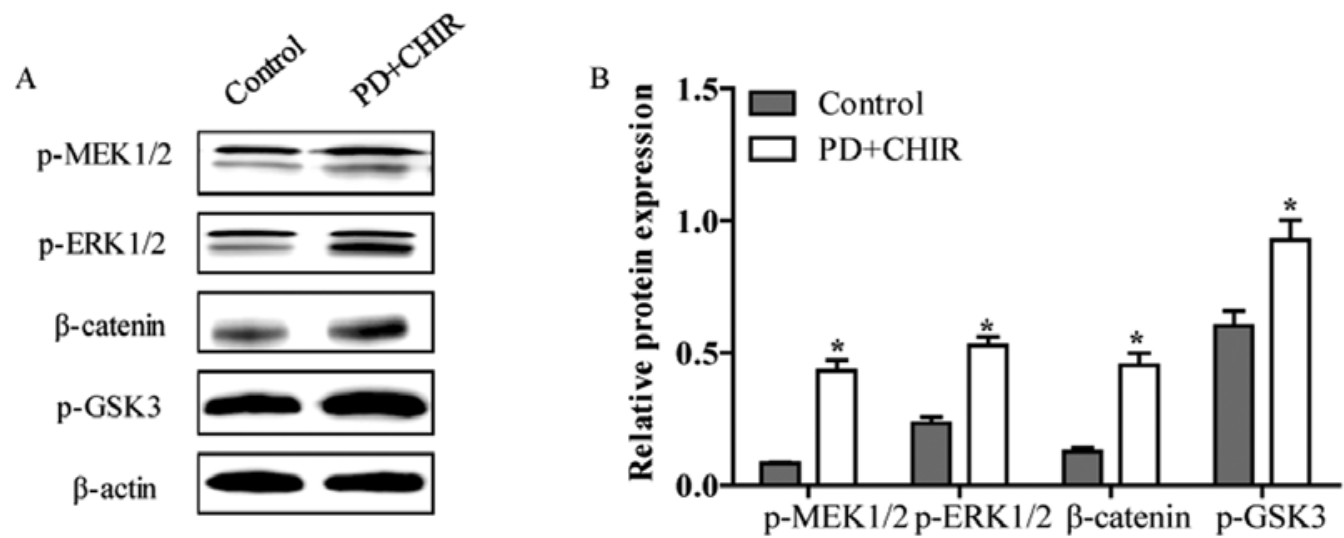

Figure 5. CHIR and PD activate Wnt and MEK signaling pathways. (A) Relative protein expression levels of p-MEK1/2, p-ERK1/2, $\beta$-catenin and p-GSK3 in human mammary epithelial cells cells following treatment with CHIR and PD detected by western blot analysis. (B) Relative quantification of western blot analysis is presented in bar charts. All assays were performed in duplicates and data are presented as the mean \pm standard deviation. ${ }^{*} \mathrm{P}<0.05$ vs. control group. PD, PD184352; CHIR, CHIR99021; MEK, mitogen-activated kinase kinase; ERK, extracellular-signal regulated kinase; GSK, glycogen synthase kinase 3; p-, phosphorylated.

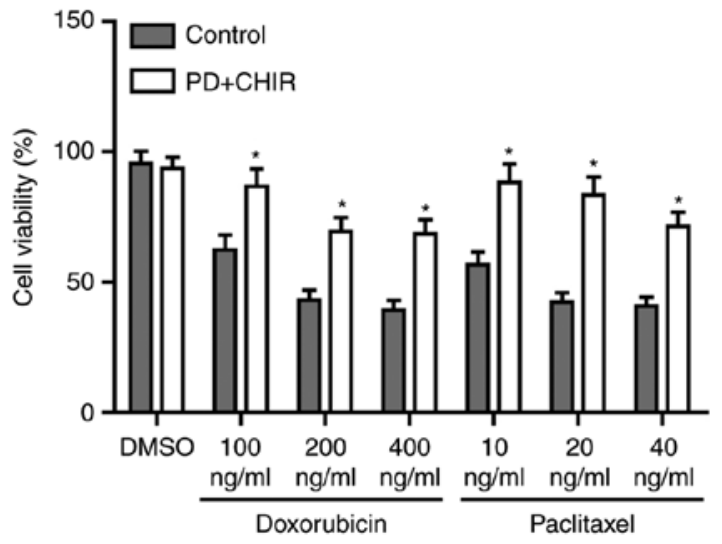

Figure 6. Human mammary epithelial cells treated with CHIR and PD exhibit cancer stem cell properties. Cells treated with compounds were resistant to doxorubicin and paclitaxel. All assays were performed in duplicates and data are presented as the mean \pm standard deviation. ${ }^{*} \mathrm{P}<0.05$ vs. control group. PD, PD184352; CHIR, CHIR99021.

contrast, the mRNA expression levels of vimentin were low in the control cells, and significantly increased following treatment with CHIR and PD for 24 and $48 \mathrm{~h}$, with the expression almost doubling after $48 \mathrm{~h}(\mathrm{P}<0.05$; Fig. $4 \mathrm{~A}$ and $\mathrm{C}$; right panel). Other mesenchymal markers, Twist and $\alpha$-smooth muscle actin ( $\alpha$-SMA), were also examined; the mRNA expression levels of Twist and $\alpha$-SMA also exhibit a significant increase at 24 and $48 \mathrm{~h}$ post-treatment compared with the control cells $(\mathrm{P}<0.05)$ and the expression levels at $48 \mathrm{~h}$ were double that at $24 \mathrm{~h}$ (Fig. 4D and E).

CHIR and PD activate the Wnt and MEK signaling pathway. The mechanisms of how CHIR and PD accelerated the cell cycle and reduced cell apoptosis in HMLE cells were examined. The relative protein levels of p-MEK1/2, p-ERK1/2, $\beta$-catenin and p-GSK3 were determined by western blot analysis. The relative protein levels of p-MEK1/2, p-ERK1/2, $\beta$-catenin and p-GSK3 were significantly increased in HMLE cells following treatment with CHIR and PD compared with the control cells $(\mathrm{P}<0.05$; Fig. $5 \mathrm{~A}$ and $\mathrm{B})$. This suggested that
Table I. Tumor incidence in mice injected with increasing dilutions of cells.

\begin{tabular}{lcc}
\hline Cells injected & Control & PD+CHIR \\
\hline $1 \times 10^{6}$ & $4 / 4$ & $4 / 4$ \\
$1 \times 10^{5}$ & $3 / 4$ & $4 / 4$ \\
$1 \times 10^{4}$ & $1 / 4$ & $4 / 4$ \\
$1 \times 10^{3}$ & $0 / 4$ & $4 / 4$ \\
\hline
\end{tabular}

PD, PD184352; CHIR, CHIR99021.

CHIR and PD-induced proliferation, invasion and migration and the inhibition of cell apoptosis may be associated with the activation of the Wnt and MEK signaling pathway.

Cells treated with CHIR and PD demonstrate the properties of CSCs. To investigate whether cells treated with CHIR and PD exhibit CSC properties, cells were treated with serial dilutions doxorubicin or paclitaxel for $24 \mathrm{~h}$. The percentage of drug resistant cells was significantly increased by CHIR and PD compared with untreated control cells $(\mathrm{P}<0.05$; Fig. 6). To determine the tumor initiation ability of the induced HMLE cells, SCID mice were injected with $10^{3}, 10^{4}$, $10^{5}$ and $10^{6}$ cells. Tumors formed in all of the mice injected with CHIR and PD-treated cells, whereas $10^{4}$ of the control cells were required to initiate tumor formation and only $75 \%$ of mice formed tumors when injected $10^{5}$ with control cells (Table I).

Cells treated with CHIR and PD promotes tumor growth in vivo. To further investigate the effects of CHIR and PD in vivo, $1 \times 10^{3} \mathrm{HMLE}$ cells were injected into SCID mice. To evaluate tumor growth, the length and width of subcutaneous tumors were measured every 7 days post-inoculation. Progressive solid tumor types were observed in all mice. The volume of the tumors generated from CHIR and PD-treated HMLE cells was significantly higher compared with those produced 
A

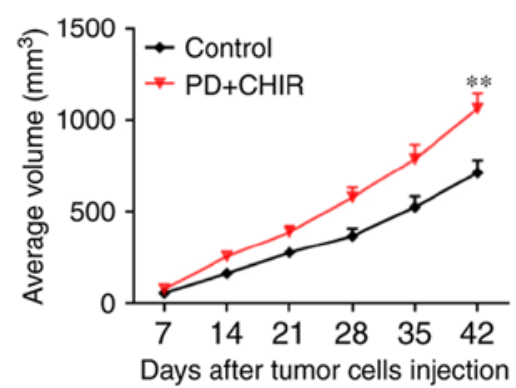

B

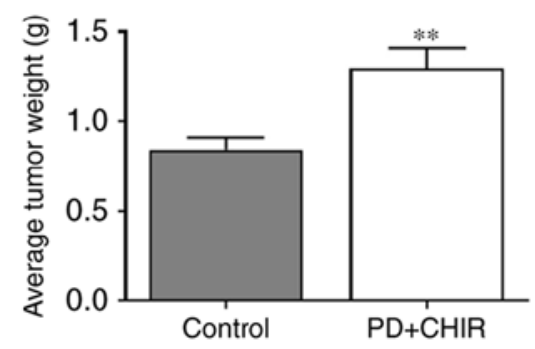

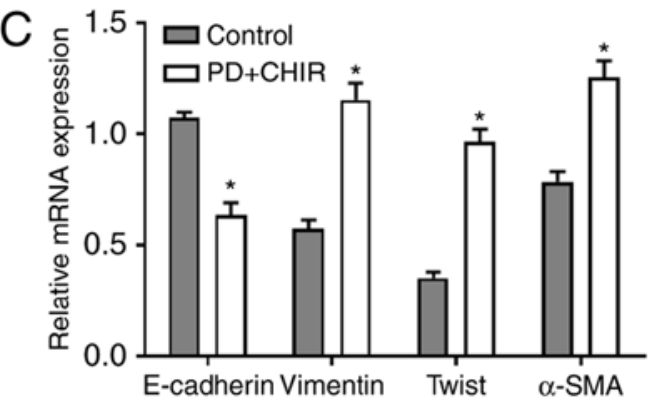

Figure 7. Treatment of cells with CHIR and PD promotes tumor growth in vivo. (A) Subcutaneous tumor mean volume following treatment with CHIR and PD. (B) Orthotopic tumor weights at 42 days post-inoculation. (C) mRNA expression levels of E-cadherin, vimentin, Twist and $\alpha$-SMA in tumor tissues were determined by reverse transcription-quantitative polymerase chain reaction analysis. All assays were performed in duplicates and data are presented as the mean \pm standard deviation. ${ }^{*} \mathrm{P}<0.05$ and ${ }^{* *} \mathrm{P}<0.01$ vs. control group. PD, PD184352; CHIR, CHIR99021; $\alpha$-SMA, $\alpha$-smooth muscle actin.

from control HMLE cells $(\mathrm{P}<0.01$; Fig. 7A). The mean tumor weights (mean \pm standard deviation) of subcutaneous tumors were as follows: CHIR and PD group, $1.316 \pm 0.235 \mathrm{~g}$, and the control group, $0.823 \pm 0.124 \mathrm{~g}$. The weight of tumors from the CHIR and PD group were significantly higher compared with the control group $(\mathrm{P}<0.01$; Fig. 7B). Additionally, the relative mRNA expression of vimentin, Twist and $\alpha$-SMA were significantly higher in the tumor tissue of the CHIR and PD group compared with the control group, and the relative mRNA expression of E-cadherin was significantly lower in the CHIR and PD group compared with that in the control group $(\mathrm{P}<0.05$; Fig. $7 \mathrm{C})$. The data suggested that the treatment of HMLE cells with CHIR and PD promoted the growth of HMLE-engrafted tumor types in vivo.

\section{Discussion}

To understand the effects of CSCs on tumor initiation and therapeutic resistance $(17,18)$, improved methods of isolation and cell expansion in vitro need to be developed. Although CSCs may be distinguished via certain cell surface markers in a variety of tumor types, cancer cells that are negative for markers may also exhibit a proliferative CSC phenotype (19). The results of previous studies have indicated that cautions should be taken when using surface markers to identify CSCs due to the phenotypic plasticity of tumor cells $(19,20)$. CHIR is implicated in the self-renewal of HMLE cells, activating canonical Wnt signaling $(8,9)$. PD is a small inhibitor of MEK that has been demonstrated to suppress HMLE cell proliferation (10). However, in the present study, non-stem cancer cells were co-treated with an GSK3 inhibitor (CHIR) and an MEK inhibitor (PD). The treated cells exhibited increased expression of CSC markers, increased tumorigenicity and therapeutic resistance. The observations of previous studies indicated, at least in part, the potential to rapidly and effectively generate CSCs by stimulation cells with specific molecules (21-23).

Previous studies have suggested that GSK3 serves an important function in various cellular processes, including mediating signaling downstream of Wnt, fibroblast growth factor (FGF) and Hedgehog during the progression of cancer $(24,25)$. Upregulation of Wnt ligands, induced by blocking GSK3, bind to the frizzled/low-density-lipopro- tein-related protein co-receptor complex, which results in the stabilization and nuclear translocation of $\beta$-catenin. $\beta$-catenin functions as a powerful trans-activator of T-cell factor/lymphoid enhancer factor transcription factors, which regulate important downstream target genes that promote cell proliferation, differentiation and tissue development. The MEK signaling pathway comprises several key signaling components and phosphorylation events that have important roles in tumorigenesis $(26,27)$. These activated kinases regulate cell growth, differentiation, proliferation and migration (28). Notably, in the present study, cancer cells treated with CHIR and PD to inhibit MEK signaling proliferated faster compared with the control cells. One potential explanation for this is that CHIR not only inhibits the Wnt signaling pathway, but also engages in cross talk with FGF signaling to enhance the expansion (29). In addition, previous studies have reported that there is bidirectional crosstalk between ERK and Wnt signaling pathways $(29,30)$. Following the inhibition of MEK using CHIR, the accumulation of $\beta$-catenin resulted in the activation of ERK and stimulated the upregulation of oncogenes, including c-myc and Ras $(31,32)$.

EMT serves a critical role in the acquisition of malignant traits by carcinoma cells, particularly during the process of metastasis $(32,33)$. During EMT, epithelial cells lose cell-cell junctions and polarity, resulting in cells exhibiting an enhanced migratory, mesenchymal cell phenotype (34). In the present study, treatment with CHIR and PD induced the specific molecular changes that are markers of EMT, including the downregulation of E-cadherin expression, and the upregulation of Twist and vimentin expression. In addition, studies revealed mammosphere cultures formed and the tumors observed in SCID mice prevented the success of the transition and capturing of CSCs $(35,36)$. These results demonstrated that EMT is an important feature of CSCs. However, Andarawewa et al (37) reported that MEK/ERK activation induced by ionizing radiation co-operated with transforming growth factor- $\beta 1$ to induce EMT in normal mammary epithelial cells, and the inhibition of MAPK/ERK activity notably prevented the downregulation of E-cadherin protein levels and restored cell-cell interaction. Sineva and Pospelov (38) additionally reported that GSK3 inhibition affected the proliferation rate of stem cells by increasing the accumulation of cells in the G1 phase. Notably, in the present study, the treated cancer cells exhibited the 
increased expression of CSC surface markers, various CSC properties and an increase in proliferation compared with the untreated control cells. One of the potential mechanisms involved in the observed aforementioned effects is that the compounds reduce $\beta$-catenin/E-cadherin-mediated adhesion, rather than the $\beta$-catenin-dependent transcription of EMT- or cell cycle-associated genes. However, the underlying molecular mechanisms of these effects remain poorly understood.

Collectively, the results of the present study demonstrated that the co-stimulation of cancer cells with PD and CHIR efficiently generated CSCs. Notably, these results also indicated that the process of inducing differentiated breast epithelial tumor cells is sufficient to promote the initiation and establishment of a tumor. The mechanisms underlying the observed effects remain largely unknown and require further investigation.

\section{Acknowledgements}

Not applicable.

\section{Funding}

No funding was received.

\section{Availability of data and materials}

The datasets used and/or analyzed during the current study are available from the corresponding author on reasonable request.

\section{Authors' contributions}

ZM and SL designed the present study. SL, LG, WQ and CL performed the experiments. SL analyzed the data. All authors read and approved the final manuscript.

\section{Ethics approval and consent to participate}

All animal experiments were ethically approved by the Research Ethics Committee of Third Military Medical University (Chongqing, China).

\section{Patient consent for publication}

Not applicable.

\section{Competing interest}

The authors declare that they have no competing interests.

\section{References}

1. Reya T, Morrison SJ, Clarke MF and Weissman IL: Stem cells, cancer, and cancer stem cells. Nature 414: 105-111, 2001.

2. Visvader JE and Lindeman GJ: Cancer stem cells in solid tumours: Accumulating evidence and unresolved questions. Nat Rev Cancer 8: 755-768, 2008.

3. Dalerba P, Cho RW and Clarke MF: Cancer stem cells: Models and concepts. Annu Rev Med 58: 267-284, 2007.

4. Rowehl RA, Crawford H, Dufour A, Ju J and Botchkina GI: Genomic analysis of prostate cancer stem cells isolated from a highly metastatic cell line. Cancer Genomics Proteomics 5: 301-310, 2008.
5. Abraham BK, Justenhoven C, Pesch B, Harth V, Weirich G, Baisch C, Rabstein S, Ko YD, Brüning T, Fischer HP, et al: Investigation of genetic variants of genes of the hemochromatosis pathway and their role in breast cancer. Cancer Epidemiol Biomarkers Prev 14: 1102-1107, 2005.

6. Hill RP and Perris R: 'Destemming' cancer stem cells. J Natl Cancer Inst 99: 1435-1440, 2007.

7. Zhu QS, Rosenblatt K, Huang KL, Lahat G, Brobey R, Bolshakov S, Nguyen T, Ding Z, Belousov R, Bill K, et al: Vimentin is a novel AKT1 target mediating motility and invasion. Oncogene 30: 457-470, 2011.

8. An WF, Germain AR, Bishop JA, Nag PP, Metkar S, Ketterman J, Walk M, Weiwer M, Liu X, Patnaik D, et al: Discovery of potent and highly selective inhibitors of GSK3b, 2010.

9. Reya T and Clevers H: Wnt signalling in stem cells and cancer. Nature 434: 843-850, 2005

10. Pellicano F, Simara P, Sinclair A, Helgason GV, Copland M, Grant S and Holyoake TL: The MEK inhibitor PD184352 enhances BMS-214662-induced apoptosis in CD34 ${ }^{+}$CML stem/progenitor cells. Leukemia 25: 1159-1167, 2011.

11. Li L, Bennett SA and Wang L: Role of E-cadherin and other cell adhesion molecules in survival and differentiation of human pluripotent stem cells. Cell Adh Migr 6: 59-70, 2012.

12. Satelli A and Li S: Vimentin in cancer and its potential as a molecular target for cancer therapy. Cell Mol Life Sci 68: 3033-3046, 2011.

13. Livak KJ and Schmittgen TD: Analysis of relative gene expression data using real-time quantitative PCR and the 2(-Delta Delta C(T)) method. Methods 25: 402-408, 2001.

14. Kawano K, Efferson CL, Peoples GE, Carter D, Tsuda N, Murray JL and Ioannides CG: Sensitivity of undifferentiated, high-TCR density CD8 ${ }^{+}$cells to methylene groups appended to tumor antigen determines their differentiation or death. Cancer Res 65: 2930-2937, 2005.

15. Kruger NJ: The Bradford method for protein quantitation. In: Methods in Molecular Biology: Basic Protein and Peptide Potocols. Chapter 32. Walker JM (ed). Humana Press, Totowa, NJ, pp9-15, 1994.

16. Gupta PB, Onder TT, Jiang G, Tao K, Kuperwasser C, Weinberg RA and Lander ES: Identification of selective inhibitors of cancer stem cells by high-throughput screening. Cell 138: 645-659, 2009.

17. Vinogradov $S$ and Wei X: Cancer stem cells and drug resistance: The potential of nanomedicine. Nanomedicine (Lond) 7: 597-615, 2012.

18. Facompre N, Nakagawa H, Herlyn M and Basu D: Stem-like cells and therapy resistance in squamous cell carcinomas. Adv Pharmacol 65: 235-265, 2012.

19. Huang SD, Yuan Y, Tang H, Liu XH, Fu CG, Cheng HZ, Bi JW, Yu YW, Gong DJ, Zhang W, et al: Tumor cells positive and negative for the common cancer stem cell markers are capable of initiating tumor growth and generating both progenies. PLoS One 8: e54579, 2013.

20. Jaggupilli A and Elkord E: Significance of CD44 and CD24 as cancer stem cell markers: An enduring ambiguity. Clin Dev Immunol 2012: 708036, 2012.

21. Li Y, Wang L, Pappan L, Galliher-Beckley A and Shi J: IL-1 $\beta$ promotes stemness and invasiveness of colon cancer cells through Zeb1 activation. Mol Cancer 11: 87, 2012.

22. Wang ZL, Fan ZQ, Jiang HD and Qu JM: Selective Cox-2 inhibitor celecoxib induces epithelial-mesenchymal transition in human lung cancer cells via activating MEK-ERK signaling. Carcinogenesis 34: 638-646, 2013.

23. Xie G, Yao Q, Liu Y, Du S, Liu A, Guo Z, Sun A, Ruan J, Chen L, Ye $C$ and Yuan Y: IL-6-induced epithelial-mesenchymal transition promotes the generation of breast cancer stem-like cells analogous to mammosphere cultures. Int J Oncol 40: 1171-1179, 2012.

24. Hur EM and Zhou FQ: GSK3 signalling in neural development. Nat Rev Neurosci 11: 539-551, 2010.

25. Fisar Z and Hroudová J: Intracellular signalling pathways and mood disorders. Folia Biol (Praha) 56: 135-148, 2010.

26. Zou Y, Liu FY, Wu J, Wan L, Fang SF, Zhang ZY, Luo Y, Chen MH, Huang MZ, He M and Huang OP: Mutational analysis of the RAS/RAF/MEK/ERK signaling pathway in 260 Han Chinese patients with cervical carcinoma. Oncol Lett 14: 2427-2431, 2017.

27. Xiong Y, Huang F, Li X, Chen Z, Feng D, Jiang H, Chen W and Zhang X: CCL21/CCR7 interaction promotes cellular migration and invasion via modulation of the MEK/ERK1/2 signaling pathway and correlates with lymphatic metastatic spread and poor prognosis in urinary bladder cancer. Int J Oncol 51: 75-90, 2017. 
28. Santarpia L, Lippman SM and El-Naggar AK: Targeting the MAPK-RAS-RAF signaling pathway in cancer therapy. Expert Opin Ther Targets 16: 103-119, 2012.

29. Singh AM, Reynolds D, Cliff T, Ohtsuka S, Mattheyses AL, Sun Y, Menendez L, Kulik M and Dalton S: Signaling network crosstalk in human pluripotent cells: A Smad2/3-regulated switch that controls the balance between self-renewal and differentiation. Cell Stem Cell 10: 312-326, 2012.

30. Gong K, Zhou F, Huang H, Gong Y and Zhang L: Suppression of GSK $3 \beta$ by ERK mediates lipopolysaccharide induced cell migration in macrophage through $\beta$-catenin signaling. Protein Cell 3: 762-768, 2012.

31. Sears R, Nuckolls F, Haura E, Taya Y, Tamai K and Nevins JR: Multiple Ras-dependent phosphorylation pathways regulate Myc protein stability. Genes Dev 14: 2501-2514, 2000.

32. Dave B, Mittal V, Tan NM and Chang JC: Epithelial-mesenchymal transition, cancer stem cells and treatment resistance. Breast Cancer Res 14: 202, 2012.

33. Wang Y and Zhou BP: Epithelial-mesenchymal transition in breast cancer progression and metastasis. Chin J Cancer 30: 603-611, 2011

34. Xiang J, Fu X, Ran W and Wang Z: Grhl2 reduces invasion and migration through inhibition of TGF $\beta$-induced EMT in gastric cancer. Oncogenesis 6: e284, 2017.
35. Mani SA, Guo W, Liao MJ, Eaton EN, Ayyanan A, Zhou AY, Brooks M, Reinhard F, Zhang CC, Shipitsin M, et al: The epithelial-mesenchymal transition generates cells with properties of stem cells. Cell 133: 704-715, 2008.

36. Phillips TM, McBride WH and Pajonk F: The response of CD24(-/low)/CD44+ breast cancer-initiating cells to radiation. J Natl Cancer Inst 98: 1777-1785, 2006.

37. Andarawewa KL, Erickson AC, Chou WS, Costes SV, Gascard P, Mott JD, Bissell MJ and Barcellos-Hoff MH: Ionizing radiation predisposes nonmalignant human mammary epithelial cells to undergo transforming growth factor beta induced epithelial to mesenchymal transition. Cancer Res 67: 8662-8670, 2007.

38. Sineva GS and Pospelov VA: Inhibition of GSK3beta enhances both adhesive and signalling activities of beta-catenin in mouse embryonic stem cells. Biol Cell 102: 549-564, 2012.

This work is licensed under a Creative Commons Attribution-NonCommercial-NoDerivatives 4.0 International (CC BY-NC-ND 4.0) License. 